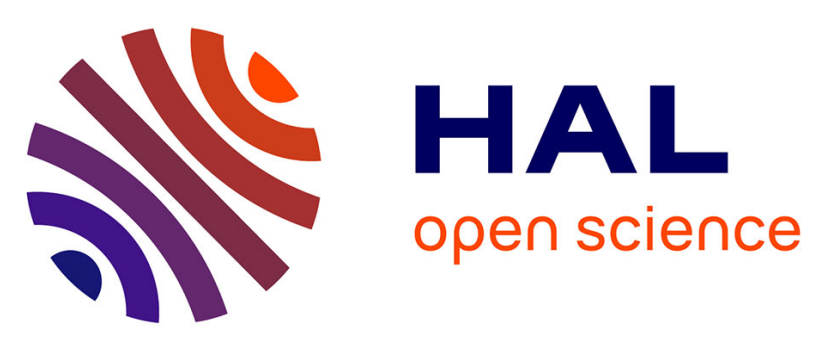

\title{
Pain, miel et poisson : exégèse patristique des aliments consommés après la résurrection
}

\author{
Matthieu Cassin
}

\section{To cite this version:}

Matthieu Cassin. Pain, miel et poisson: exégèse patristique des aliments consommés après la résurrection. Revue d'études augustiniennes et patristiques, 2019, 65 (2), pp.287-305. 10.1484/J.REA.5.120341 . halshs-02512357

\section{HAL Id: halshs-02512357 https://shs.hal.science/halshs-02512357}

Submitted on 19 Mar 2020

HAL is a multi-disciplinary open access archive for the deposit and dissemination of scientific research documents, whether they are published or not. The documents may come from teaching and research institutions in France or abroad, or from public or private research centers.
L'archive ouverte pluridisciplinaire HAL, est destinée au dépôt et à la diffusion de documents scientifiques de niveau recherche, publiés ou non, émanant des établissements d'enseignement et de recherche français ou étrangers, des laboratoires publics ou privés. 


\section{Pain, miel et poisson : exégèse patristique des aliments consommés après la résurrection}

Que mangea le Christ après la résurrection? La question peut sembler pour le moins anecdotique : l'important demeure qu'il ait mangé, et c'est bien le point que la plupart des auteurs patristiques ont commenté, soulignant ainsi la réalité de la résurrection charnelle du Christ. Cependant, il n'est pas sans intérêt de mener un petit inventaire des aliments consommés par le ressuscité, et des variations textuelles à ce sujet. Dans les évangiles de Matthieu comme de Marc, les choses sont simples : il n'est pas question de repas du ressuscité. Dans ceux de Luc et de Jean, en revanche, les choses paraissent plus complexes. En Lc 24, 30, dans l'épisode d'Emmaüs, il est fait mention du pain pris et rompu ( $\lambda \alpha \beta \omega \mathrm{v}$

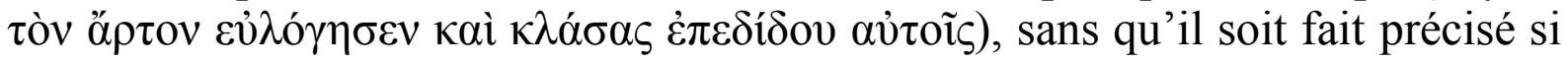
le Christ a effectivement mangé, non plus que les disciples, qui comprennent, et partent, apparemment sans se nourrir :

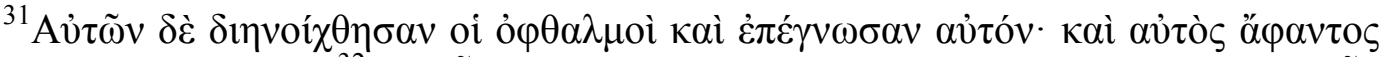

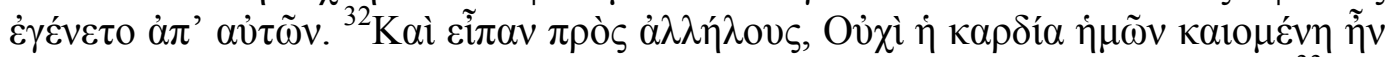

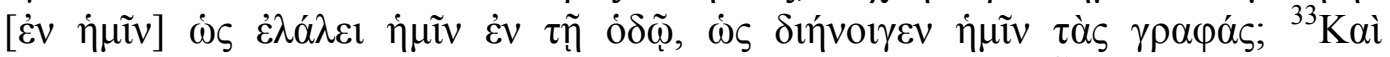

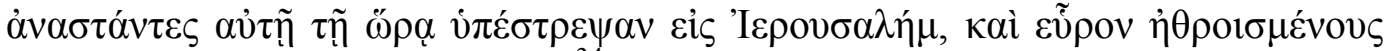

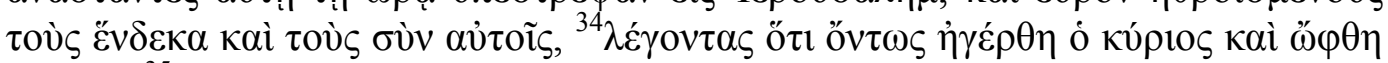

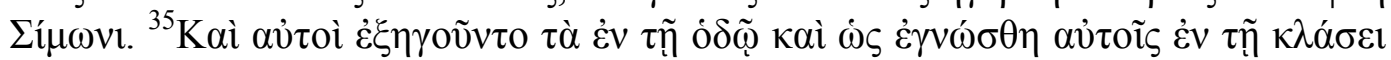

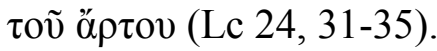

Dans l'épisode de Lc 24, 36-49, lors de l'apparition du Christ aux disciples réunis à Jérusalem, y compris les deux disciples d'Emmaüs, Jésus mange du

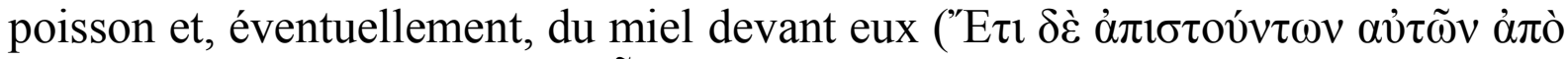

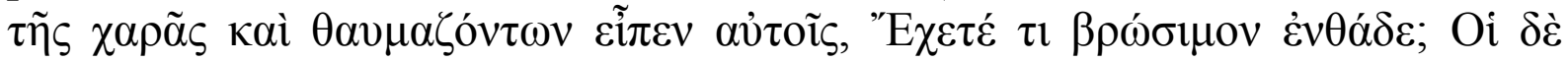

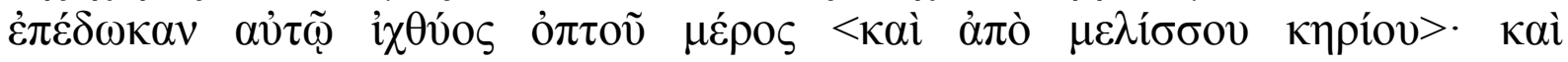

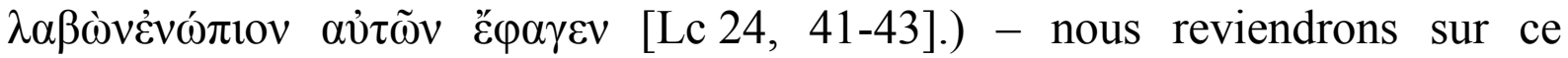
passage, qui sera au cœur de l'étude que nous proposons ici.

Enfin, dans l'évangile de Jean, après l'épisode de la pêche des 153 poissons au matin sur le Lac, on trouve une longue description d'un repas matinal au bord de ce même lac, où il n'est cependant pas explicitement dit que Jésus, qui fait ici office de cuisinier, ait mangé lui-même, alors qu'il invite ses disciples à le faire : 


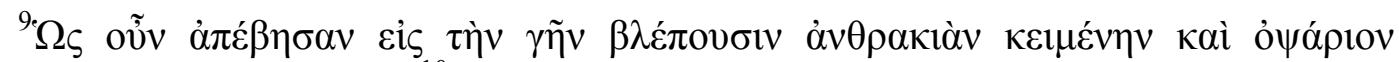

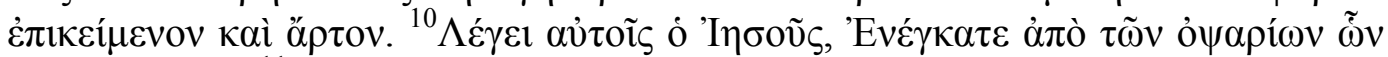

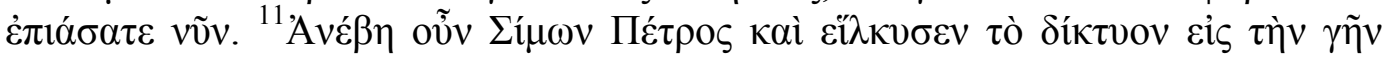

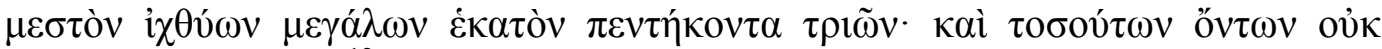

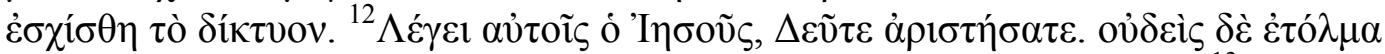

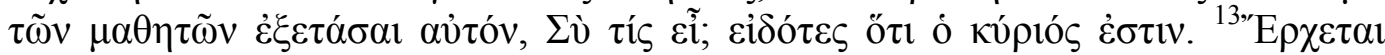

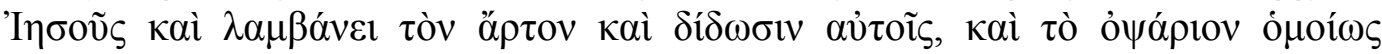
(Jn 21, 9-13).

Dans ce dernier cas, ce sont donc du pain et du poisson qui sont préparés par le Christ ressuscité et offerts à ses disciples. Non seulement les ingrédients diffèrent de ceux que l'on trouve dans le texte lucanien, mais surtout, en Jean c'est le Christ qui cuisine, alors qu'en Luc, il demande de la nourriture aux disciples.

La liste des aliments qui ont été explicitement consommés est réduite : du poisson et du pain (Jn), du poisson et éventuellement du miel (Lc) ; il faut y ajouter le pain dans l'épisode d'Emmaüs, dont on ne sait s'il resta sur la table quand le Christ disparut à leurs yeux. La plupart des explications patristiques de ces passages ne s'attardent pas outre mesure sur les aliments consommés et soulignent surtout que le Christ a mangé 1 . Il est cependant quelques cas dans lesquels les ingrédients ont retenu l'attention des commentateurs ${ }^{2}$. Nous nous arrêterons ici en particulier sur un point, qui a en outre l'intérêt d'être lié d'une part à une variante du texte néotestamentaire - la mention du rayon de miel en Lc 24, 42, en accompagnement du poisson - et d'autre part à la conflation des deux repas principaux de Luc, à Jérusalem, et de Jean, au bord du lac. Après avoir présenté d'abord un rapide état de la question sur la variante de Lc 24, 42, on étudiera trois textes qui témoignent de l'interprétation, en Palestine et en Cappadoce, à la fin du $4^{\mathrm{e}}$ siècle, de cette variante de Lc 24,42 , en lien avec un verset du Cantique des cantiques $(\mathrm{Ct} 5,1 \mathrm{c})$. L'un de ces textes va même jusqu'à fusionner les récits de Luc et de Jean, en rapprochant non plus poisson et miel, mais pain et miel. Dans un dernier temps, on présentera rapidement d'autres

1 Il faut mentionner également le témoignage d'IGNACE D'ANTIOCHE, Lettre aux Smyrniotes, III, 1-3 (éd. F. X. FunK, K. BIhlmEYER, Die Apostolischen Väter, Tübingen, 1970), qui rappelle, comme preuve de la résurrection du Christ en la chair, qu'il a «mangé et bu » avec ses disciples après la résurrection, en référence à Ac 10, 41. Ce verset - qui transmet une tradition légèrement différente de celle des évangiles, où il n'est pas question de boisson, dans les repas du ressuscité - est cependant très rarement cité, et jamais, hors Ignace d'Antioche, avant Jean Chrysostome et Théodoret de Cyr (le renvoi de Biblindex à la Didascalia apostolorum, 20, possible, n'est pas assuré, dans la mesure où il y est seulement question de manger avec le ressuscité, non de boire avec lui).

2 On relèvera pour mémoire l'article de C. VoGEL, «Le repas sacré au poisson chez les Chrétiens », Revue des sciences religieuses, 40, 1966, p. 1-26, qui mentionne la variante de Lc 24, 42, à propos de son étude sur les repas avec poisson, mais sans l'étudier en détail, puisque son intérêt porte sur l'ingrédient présent dans tous les témoins, le poisson. Voir également A. MacGowan, Ascetic Eucharists : Food and Drink in Early Christian Ritual Meals (Oxford Early Christian Studies), Oxford, 1999, p. 127-128. 
témoignages patristiques sur la variante de Lc 24,42 , afin de tenter de cerner sinon son origine et sa date, du moins de la mieux situer dans le temps et l'espace, sans prétendre cependant épuiser le dossier pour les interprétations ultérieures du poisson et du miel, qui deviennent nombreuses à partir du moment où le texte long devient le texte communément admis 3 .

$$
\text { I. - LC } 24,42
$$

Quel est le texte exact de Lc 24, 42 ? Ce que nous considérons, avec nos yeux modernes, académiques et occidentaux, comme une variante du texte biblique la mention du rayon de miel - est en fait le texte byzantin reçu et celui de la Vulgate ${ }^{4}$. Le texte de l'édition courante, la $28^{\mathrm{e}}$ édition Nestle-Aland, est le

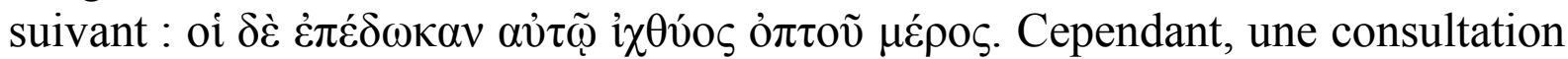
tant de l'apparat de cette édition que des collations publiées dans le volume d'analyse pour l'évangile de Luc par l'institut de Münster confirme rapidement que, si ce texte court a pour lui de très bons témoins $\left(\mathrm{P}^{75} \times \mathrm{A}\right.$ B D L W 041579 $\left.10792411^{\mathrm{pc}}\right)$, il reflète cependant une forme tout à fait minoritaire, qui correspond au texte alexandrin. Dans l'immense majorité des témoins, on trouve

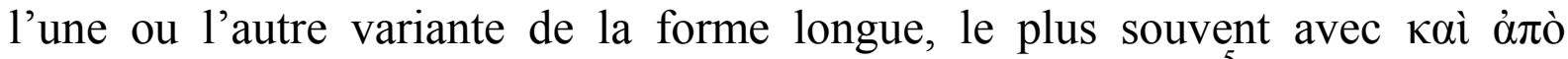

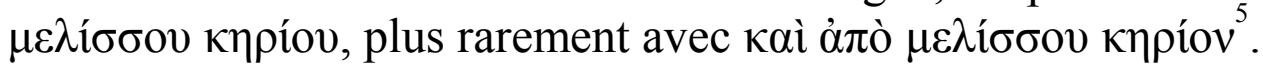

Qu'en est-il des traductions anciennes? La plupart n'ont pas la leçon longue : en syriaque, elle est absente de la version sinaïtique (mi- $4^{\mathrm{e}} \mathrm{s}$. ?) de même que de

3 B. CHERUBINI a consacré en 2006 sa tesi di laurea à la variante de Lc 24, 42 ("Mangiò pesce e miele": un'antica tradizione sul Risorto, Università degli studi " Roma Tre », Roma, 2006) - je remercie vivement l'auteur de m'avoir communiqué son travail, ainsi qu'AnneCatherine Baudoin qui a bien voulu nous mettre en relation. La thèse présente un recensement sommaire des manuscrits qui contiennent la variante (mais sans avoir pu utiliser le volume de collation cité infra $\mathrm{n} .{ }^{* *}$ ), et une présentation des différents passages patristiques qui sont retenus comme des témoins de cette variante longue. Contrairement à la démarche ici adoptée, les témoignages patristiques sont globalement considérés comme probants dans tous les cas, à l'exception de celui de Clément, qui n'est pas mentionné ; en outre, les textes pseudépigraphes, en particulier les Quaestiones in scripturam sacram (CPG 2860) du pseudoAthanase, sont généralement considérés comme authentiques, ce qui fausse quelque peu la perspective. Je ne renverrai pas systématiquement, dans les pages qui suivent, à ce très utile travail préliminaire, sauf lorsque je suis en désaccord direct avec l'auteur.

4 Sur ce passage lucanien, voir le commentaire et les références de F. Bovon, L'évangile selon saint Luc $(19,28-24,53)$ (Commentaire du Nouveau Testament, $2^{\mathrm{e}}$ série IIId), Genève, 2009 , p. 466. Sur la variante, voir en particulier E. NeSTLE, «The Honeycomb in Luke XXIV », Expository Time, 22, 1911, p. 567-568 ; G. D. KILPATRICK, «Luke 24:42-43 », Novum Testamentum, 28, 1986, p. 306-308 ; au contraire, G. O’Collins, « Did Jesus Eat the Fish (Luke 24:42-43)? », Gregorianum, 69, 1988, p. 65-76 non seulement ne s'intéresse pas à la variante longue, mais ne la mentionne même pas.

5 Voir E. et E. Nestle, B. et K. Aland et alii, Novum testamentum graece, Stuttgart, $2012^{28}$; K. et B. AlAnd, K. WACHTEL et K. WitTE, Text und Textwert der griechischen Handschriften des Neuen Testaments. IV, Die Synoptischen Evangelien. 3, Das Lukasevangelium. 2, Resultate der Kollation und Hauptliste sowie Ergänzungen (Arbeiten zur Neustestamentlichen Textforschung 31), Berlin, New York, 2003, p. 131-132. 
la version copte sahidique ( $3^{\mathrm{e}} \mathrm{s}$. ?); du côté latin, l'attestation est plus fluctuante : on trouve la leçon courte dans le Palatinus, e, ou le codex de Bèze (en conformité avec son texte grec); en l'absence de citation ancienne du verset, il n'est pas possible de reconstituer avec plus de sûreté les attestations de cette forme courte dans le monde latin 6 . En revanche, dans les versions plus tardives, syriaque curetonienne, peshitta et harkléenne, vulgate, et dans une partie de la tradition copte bohaïrique, on trouve la leçon longue. Du côté des témoins grecs, les témoins des textes césaréen et byzantin ont la forme longue. Il faut également mentionner le cas du Diatessaron attribué à Tatien : on sait que cette forme particulière du texte évangélique ne nous est plus connue que par des versions diverses et parfois fort tardives. On a parfois voulu trouver dans cette œuvre un témoignage en faveur de l'ancienneté de la forme longue de Lc 24, 42 ; cependant, dans la mesure où la variante n'est pas présente de manière unanime dans la tradition du Diatessaron et qu'elle apparaît surtout dans des formes tardives, il paraît pour le moins périlleux d'utiliser ce témoignage en faveur de l'ancienneté ou de la localisation de l'origine de cette variante?

Depuis quand cette version longue du verset est-elle attestée ? On a parfois voulu voir dans un passage de Clément d'Alexandrie, qui cite le verset, puis parle du miel, un témoignage de ce qu'il connaissait cette leçon ${ }^{8}$. Cependant, un examen même sommaire du texte suffit à convaincre du contraire :

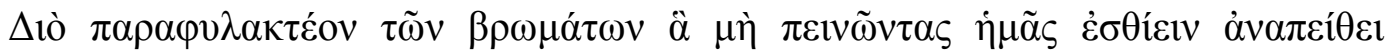

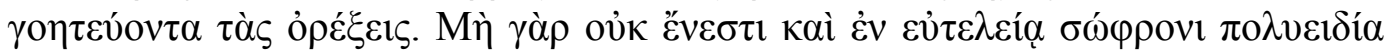

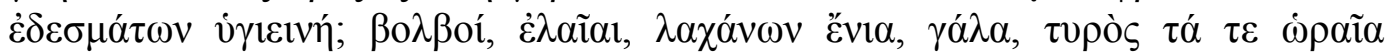

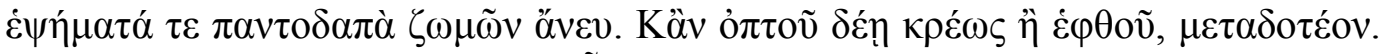

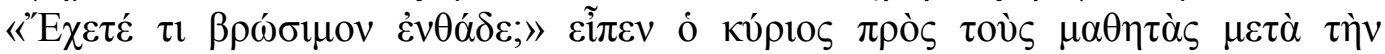

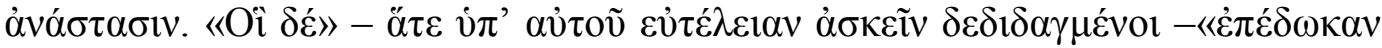

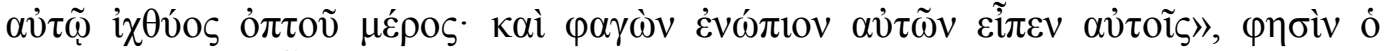
$\Lambda$

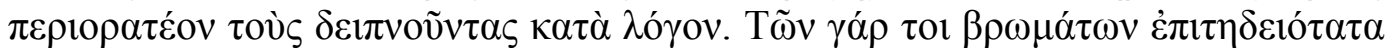

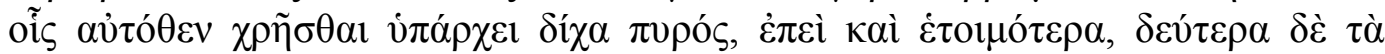
$\varepsilon \dot{\tau} \tau \varepsilon \lambda \varepsilon \dot{\sigma} \sigma \varepsilon \rho \alpha, \hat{\omega} \zeta \pi \rho \circ \varepsilon \imath \rho \dot{\kappa} \alpha \mu \varepsilon v$.

«C'est pourquoi il faut se garder des aliments qui, sans que nous ayons faim, nous induisent à manger parce qu'ils enchantent notre appétit. Est-ce qu'il n'y a pas aussi dans une simplicité modérée une saine variété de nourritures ? Des oignons, des olives, quelques légumes, du lait, du fromage, des fruits, divers

6 Enquête menée grâce à la Vetus Latina Database. On relève cependant AMBROISE DE Milan, De Cain et Abel, II, 20: in euangelio quoque assos pisces dominus Iesus manducabat, sicut scriptum est, qui est cependant une paraphrase, non une citation, et peut avoir laissé de côté le miel.

7 Voir la présentation du dossier par B. CHERUBINI, "Mangiò pesce e miele", cité n. **, p. 23-26, avec les références aux différentes versions. L'auteur conclut, avec prudence, à la présence de la forme longue dans le Diatessaron originel, du fait de sa présence dans un certain nombre de versions plus récentes. La conclusion paraît cependant fragile.

8 Voir par exemple M. HeImgartner, Pseudo-Justin - Über die Auferstehung, Text und Studie (PTS 54), Berlin, New York, 2001, p. 85-86, 180 n. 205. 
aliments cuits sans sauce. Et s'il faut de la viande rôtie ou bouillie, qu'on en donne! 'Avez-vous ici quelque chose à manger ?' dit le Seigneur à ses disciples après la résurrection. 'Ceux-ci' - parce qu'il leur avait appris à pratiquer la simplicité - lui donnèrent un morceau de poisson rôti ; et lui, tout en mangeant sous leurs yeux, leur dit...' les paroles qui sont dans Luc. En outre, il ne faut pas laisser sans dessert ni miel ceux qui prennent leur repas de façon raisonnable ${ }^{9}$. »

En effet, la citation de Lc 24, 42 se termine clairement, en l'absence de la mention du miel. Et il n'est question de miel (et non de rayon de miel) que dans une étape ultérieure, lorsque sont évoqués les douceurs qui terminent le repas. La citation évangélique ne vient qu'appuyer la description du plat principal, sur le modèle de celui du Christ ressuscité, modèle parfait et accompli de la nourriture convenablement mesurée, tandis que le miel intervient ensuite, pour clore le repas ${ }^{10}$. Les autres attestations anciennes qui ont été proposées de cette variante longue, en particulier un passage de Tertullien, dans le De corona (14, 4), Atquin et fauos post fella gustauit, ne paraissent pas convaincantes ${ }^{11}$ : il ne s'agit en aucun cas d'une citation du verset lucanien, mais d'une simple reprise de l'opposition traditionnelle entre fiel et miel, avec un jeu de sonorité qui vient renforcer le jeu sémantique. Ce miel peut-il être mis en relation avec la variante lucanienne? L'introduction de la mention du miel dans le texte de Luc est généralement lue comme une allusion à une pratique post-baptismale assez bien attestée, et ce très tôt : aux nouveaux baptisés étaient offerts du lait et du miel, signe de leur entrée dans la Terre promise ${ }^{12}$. Cependant, il n'est pas question de lait dans la variante lucanienne, mais seulement de miel, et ce miel est associé à du poisson. Si lien il y a, il est pour le moins restreint et indirect. Sans doute vaudrait-il mieux chercher ailleurs la source de la variante. Mais qu'en est-il en revanche des textes, en particulier latins, qui associent, ou plutôt font se succéder, fiel et miel ? Il semble qu'il s'agit là d'une interprétation qui pourrait dériver du même contexte que le précédent : au fiel de la passion (Mt 27, 34 ; cf. Mc 15, 23) répond le miel, jeu de mot sensible en latin uniquement - le fiel se

9 Clement D'Alexandrie, Pédagogue, II, 1, 15, 1-3, éd. O. StÄhlin et U. Treu (GCS), Berlin, $1972^{3}$, p. 164-165 ; trad. C. Mondésert (SC 108), Paris, 1965, p. 39.

10 On relèvera d'ailleurs que le traducteur de la collection Sources chrétiennes ne s'y est pas trompé et renvoie en note, pour la mention du miel, à la tradition platonicienne (ibidem, p. 39, n.12) : Platon, République, 372c ; Plutarque, Propos de table, IV, 644A.

11 Pour une interprétation différente du passage, qui voit en Tertullien un témoin direct de la variante longue, voir en particulier CHERUBINI, "Mangiò pesce e miele", cité n. **, p. 2930. Au contraire, on a vu depuis longtemps dans ce passage un proverbe, assez largement attesté, quoique sous des formes diverses, dans la littérature latine y compris non chrétienne : voir par exemple A. Отто, Die Sprichwörter und sprichwörtlichen Redensarten der Römer, Leipzig 1890, p. 133 (fauus, 2) et 217-218 (mel, 3).

12 Sur cette interprétation du miel, voir par exemple les éléments rassemblés par H. R. DRobner, Die drei Tage zwischen Tod und Auferstehung unseres Herrn Jesus Christus, eingeleitet, übersetzt und kommentiert (Philosophia Patrum 5), Leiden, 1982, p. 166 et n. 713, ainsi que MACGOWAN, Ascetic Eucharists, cité n. **. 
dit $\chi 0 \lambda \tilde{\eta}$ en $\operatorname{grec}^{13}$. Mais une telle opposition joue essentiellement sur une reprise des contraires, qui oppose le lait et le miel de la Terre promise au vinaigre et au fiel de la Passion. Dans tous ces cas où tout ou partie de ces deux couples sont opposés, jamais Lc 24, 42 n'est cité ou même mentionné. Il ne faut donc pas voir dans les mentions du miel en lien avec la résurrection une allusion automatique à Lc 24, 42, au contraire ; ce n'est généralement pas le cas, sauf lorsqu'il est associé au poisson. Le plus souvent, il s'agit simplement d'une lecture typologique des aliments de la Terre promise, appliqué à la résurrection.

\title{
II. - CT 5, 1 ET LC 24, 42 : EXEGESE PASCALE ET BAPTISMALE
}

Cependant, il est des cas, rares, où le miel et éventuellement le lait sont explicitement rapprochés de Lc 24, 42. Il s'agit de quatre textes, dus respectivement à Cyrille de Jérusalem et à Grégoire de Nysse. Nous en proposerons successivement l'étude.

\section{A. Cyrille de Jérusalem}

Le premier texte est une catéchèse baptismale de Cyrille de Jérusalem, qui peut être située dans les années 350, sans doute en leur début ${ }^{14}$. Cyrille y présente une exégèse typologique de l'Ancien Testament en lien avec la Passion et la résurrection du Christ. Si une telle démarche est courante dans la littérature patristique, il est en revanche beaucoup plus rare d'utiliser dans ce cadre le Cantique des cantiques, comme le fait ici largement Cyrille. Dans la catéchèse $14, \S 11$, l'évêque de Jérusalem applique, stique par stique, Ct 5, 1 à la mort et à la résurrection du Christ.

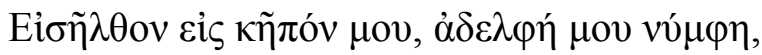

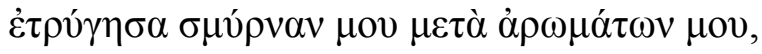

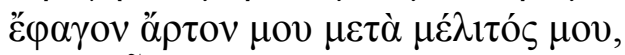

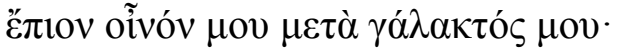

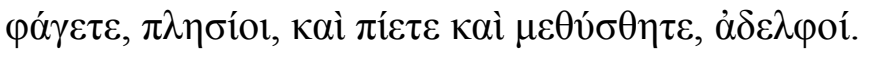 \\ « Je suis entré dans mon jardin, ma sœur, mon épouse, \\ j'ai récolté ma myrrhe avec mes aromates, \\ j'ai mangé mon pain avec mon miel, \\ j'ai bu mon vin avec mon lait. \\ Mangez, mes proches, buvez et enivrez-vous, mes frères ! ». (Ct 5,1$)$
}

13 Voir cependant Clement D'AleXandrie, Pédagogue, I, 11, 96, 2; PseudoAthanase, Homilia de passione et cruce domini (PG 28), 228, 2 ; Asterios, Hom. in Ps, 28, 6,$18 ; 29,2,19$, etc.

14 Voir S. GRIGNON, "La cohérence de la foi ». Lire les Catéchèses prébaptismales de Cyrille de Jérusalem, thèse de doctorat, Université Paris IV-Sorbonne, Paris 2003, p. 171172, et A. DovaL, "The Date of Cyril of Jerusalem's Catecheses », Journal of Theological Studies 48, 1997, p. 129-132. Voir également depuis la reconstitution de la carrière de Cyrille proposée par P. VAN Nuffelen, "The Career of Cyril of Jerusalem (c. 348-87): A Reassessment », Journal of Theological Studies 58, 2007, p. 134-146, qui ne traite cependant pas de la date des Catéchèses. 
Partant de la mention du jardin, rapproché de la tombe, Cyrille relie ensuite Ct 5, 1b et Ct 4, 14 ( $\sigma \mu v ́ \rho v \alpha \alpha \lambda \omega \theta \mu \varepsilon \tau \alpha \grave{\alpha} \pi \alpha ́ v \tau \omega v ~ \pi \rho \omega ́ \tau \omega v ~ \mu u ́ \rho \omega v$, « myrrhe et aloès et tous les principaux aromates $»)$, en en faisant un type de Nicodème et des saintes femmes lors de l'ensevelissement de Jésus. Ct 5, 1c-e est ensuite expliqué en lien avec les apparitions et les repas du ressuscité ; Lc 24, 42var. permet à l'auteur d'introduire le miel, en correspondance avec $\mathrm{Ct} 5,1 \mathrm{c}$, sans que le pain, qui n'est pas mentionné par Cyrille, apparaisse. On relèvera en revanche, dans la ligne des éléments suggérés plus haut pour le rapport entre miel et fiel, l'opposition soulignée par Cyrille entre l'amertume, avant la Passion, et la douceur, après la résurrection, appuyée sur la seule mention de Ct 5, 1c ; Lc 24, 42var n'est introduit que dans un second temps, sans reprise du thème de l'amertume et de la douceur, comme une seconde réalisation du miel de $\mathrm{Ct} 5,1 \mathrm{c}$.

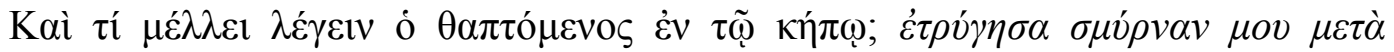

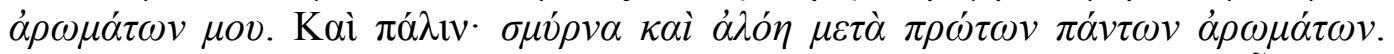

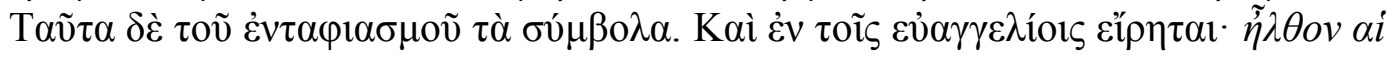

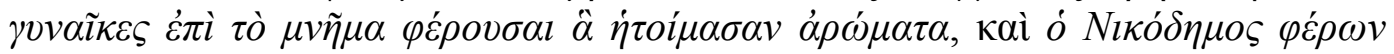

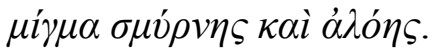

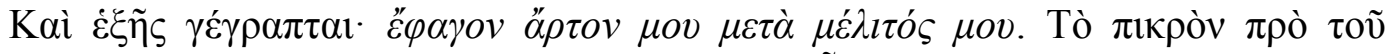

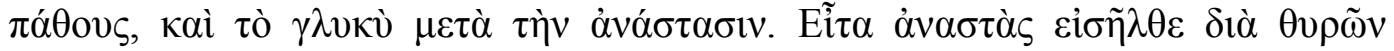

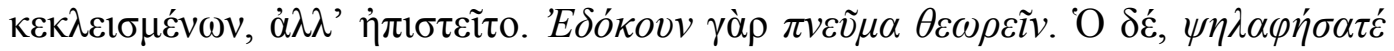

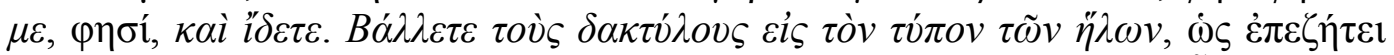

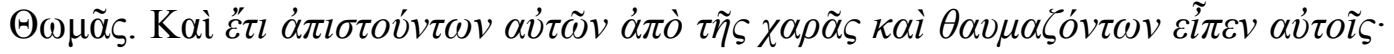

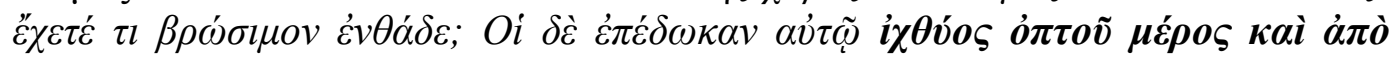

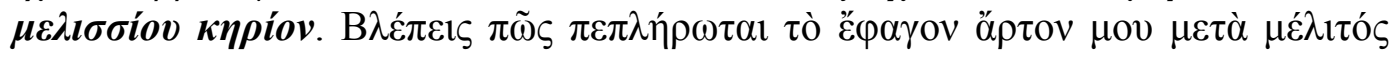

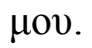

«Et que dira celui qui est enterré dans le jardin? J'ai récolté ma myrrhe avec mes aromates $(\mathrm{Ct} 5,1 \mathrm{~b})$; et encore: myrrhe et aloès, avec tous les principaux aromates $(\mathrm{Ct} 4,14)$. Voilà les symboles de l'ensevelissement. Et dans les évangiles, il est dit: Les femmes vinrent au tombeau, apportant les aromates qu'elles avaient préparées $(\operatorname{Lc} 24,1)$ et Nicodème apportant un mélange de myrrhe et d'aloès (Jn 19, 39).

Et à la suite il est écrit : J'ai mangé mon pain avec mon miel (Ct 5, 1c). L'amer, avant la passion, et le doux, après la résurrection. Puis, une fois ressuscité, il entra par des portes fermées, mais ils ne le croyaient pas. Car ils pensaient voir un esprit (Lc 24, 37). Mais touchez-moi, dit-il, et voyez (Lc 24, 39). Mettez les doigts dans les marques des clous, comme le demandait Thomas (cf. Jn 20, 25). Et comme ils ne croyaient pas encore, de joie, et s'étonnaient, il leur dit: Avez-vous ici quelque chose à manger? Et ils lui donnèrent un morceau de poisson cuit et d'un rayon de miel ( $\mathrm{Lc} 24,41-42)$. Tu vois comment est accompli J'ai mangé mon pain avec mon miel (Ct $5,1 \mathrm{c})$. 15

L'exégèse de $\mathrm{Ct} 5,1 \mathrm{c}$ se déploie donc en deux temps, d'abord une explication du miel comme la douceur de la résurrection, qui succède à l'amertume de la

15 Cyrille de Jerusalem, Catéchèses baptismales, XIV, 11 (éd. W. K. ReISChL, J. RupP, München, I-II, 1848-1860, II, p. 120). 
Passion, puis un second développement qui interprète le miel au regard de l'apparition et du repas pascal de Lc 24, 36-43. Dans cette deuxième phase, on trouve d'abord une interprétation traditionnelle du repas comme témoignage de la réalité corporelle de la résurrection du Christ, avec la reprise de Lc 24, 37 et de la peur des disciples qui croient voir un esprit. Ce n'est qu'à la toute fin de l'explication que le deuxième ingrédient du repas est rapproché de $\mathrm{Ct} 5,1 \mathrm{c}$, fermant ainsi en anneau l'interprétation. On retrouve la même exégèse dans l'Homélie sur le paralytique du même Cyrille ${ }^{16}$, qui dépend directement de la Catéchèse XIV, pour cette section, et n'apporte aucun élément nouveau17.

Il semble donc que ce soit Cyrille de Jérusalem qui ait le premier proposé de rapprocher $\mathrm{Ct} 5,1 \mathrm{c}$ et Jn 24, 42var, sans s'arrêter toutefois à l'écart entre pain, d'un côté, et poisson, de l'autre. En outre, dans ce passage, Cyrille rapproche plusieurs épisodes venant tant de Luc que de Jean, mais le rapprochement entre les récits évangéliques se fait pour le toucher, non pour les repas. L'épisode de Thomas, pris à Jean, est ajouté au fil principal, qui reste lucanien. Quelles sont les sources de Cyrille pour ce passage ? Il ne semble pas qu'elles aient pu être repérées jusqu'à présent 18 . Il ne semble pas qu'il faille chercher cette source chez Origène ou ses successeurs, dans la mesure où la seule citation de Lc 24, 42 que l'on trouve dans l'œuvre conservée d'Origène témoigne de la forme courte $^{19}$, et il en va de même chez Eusèbe de Césarée 20 .

16 Sur ce texte, voir par exemple J. W. DrIJVERs, Cyril of Jerusalem: Bishop and City (Supplements to Vigiliae Christianae 72), Leiden, 2004, p. 49-50. Sur l'interprétation du Cantique par Cyrille dans le contexte de la passion, voir M. BIELAWSKI, « Pieśń nad pieśniami w ręku Cyryla św Jerozolimskiego », Vox Patrum, 18, 1990, p. 225-242 (avec résumé en italien).

17 Cyrille de Jerusalem, Homélie sur le paralytique, 11 (ibidem, p. 416) : Tà toívvv

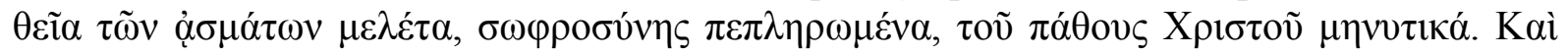

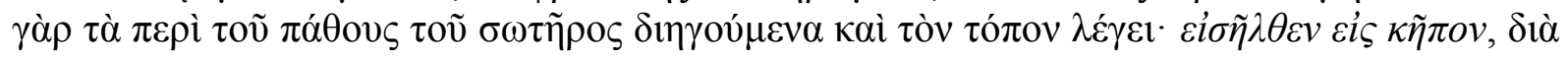

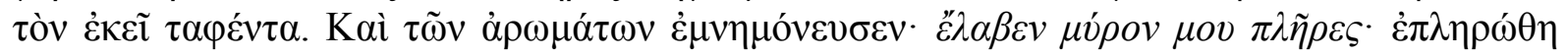

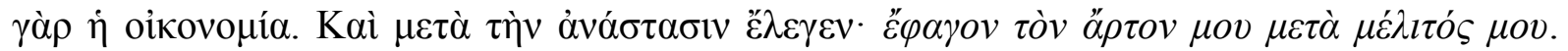

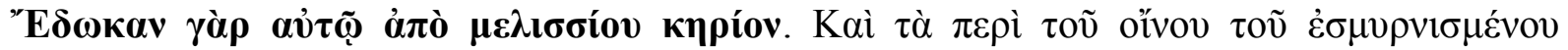

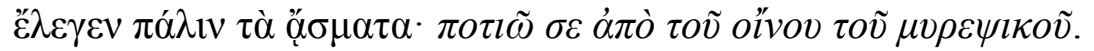

18 Pour le texte du Nouveau Testament chez Cyrille de Jérusalem, voir R. L. Mullen, The New Testament Text of Cyril of Jerusalem (The New Testament in the Greek Fathers 7), Atlanta, 1997.

19 Origène, Commentaire sur Matthieu, XI, 2 (éd. E. Klostermann [GCS 40], Leipzig,

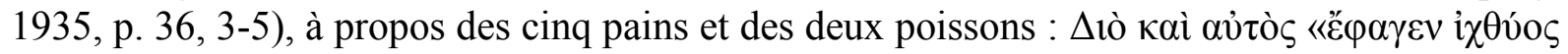

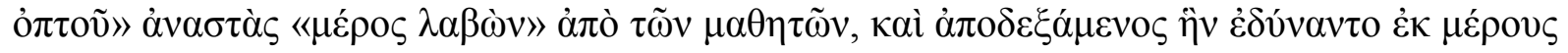

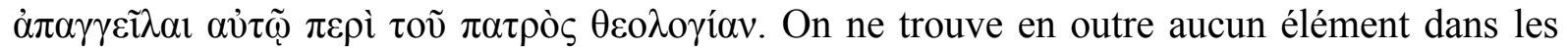
fragments origéniens sur le Cantique; voir en particulier J.-M. AuWERS, Procopii Gazaei Epitome in Canticum canticorum (Corpus christianorum, Series graeca 67), Turnhout, 2011 : pour Ct 5, 1 ne sont cités que Grégoire de Nysse et Nil d'Ancyre.

20 Du moins si le fragment des Questions à Marinos édité par A. Mai à partir de la Chaîne de Nicétas sur Luc est authentique, puisqu'il semble qu'il s'agit de l'unique occurrence du verset chez Eusèbe : Questions à Marinos, suppl. VIII (PG 22), 1001A ; voir également la 


\section{B. Grégoire de Nysse}

On retrouve un tel rapprochement entre Ct 5, 1 et Lc 24, 42 dans deux textes de Grégoire, évêque de Nysse ${ }^{21}$. Parmi ses dernières œuvres figure un commentaire du Cantique des cantiques sous forme d'une série homilétique, que l'on peut dater avec une relative certitude après 391 pour sa version écrite et diffusée, du fait des circonstances de la dédicace à Olympias, la célèbre diaconesse de Constantinople22. Dans la dixième homélie, lorsqu'il explique Ct 5, 1, Grégoire rapproche myrrhe et aromates (Ct 5, 1b) de la vie de purification. Le stique suivant, $\mathrm{Ct} 5,1 \mathrm{c}$, correspond ensuite à un niveau de nourriture plus parfait, qui est rapproché du repas proposé par les disciples à Jésus après sa résurrection, composé de pain et de miel. Enfin, Grégoire oppose $\mathrm{Ct} 5,1 \mathrm{~d}$, lait et vin, au vinaigre et au fiel de la passion.

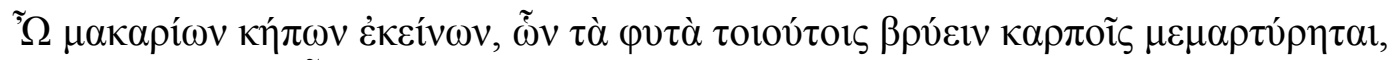

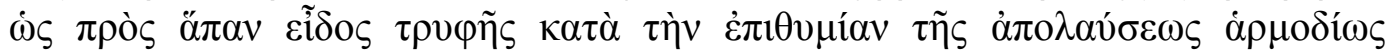

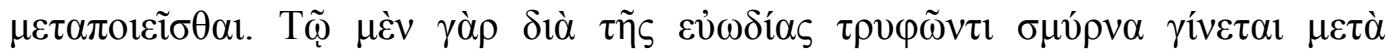

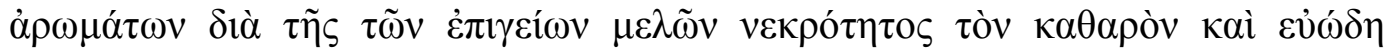

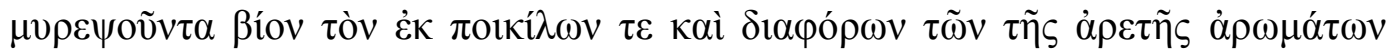

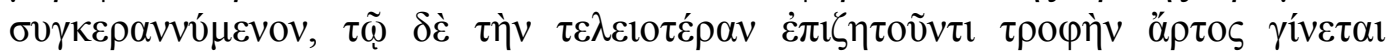

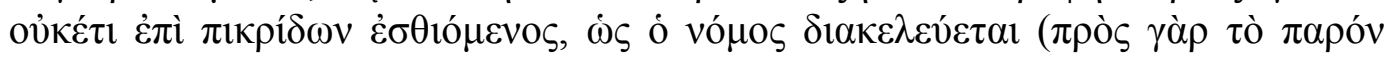

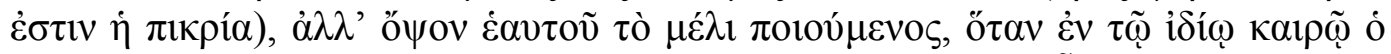

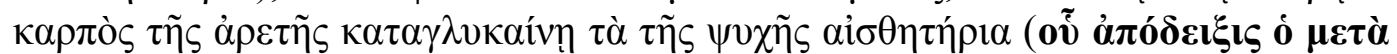

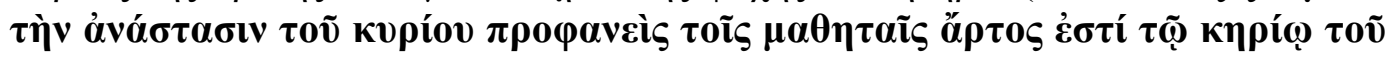

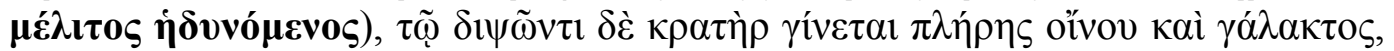

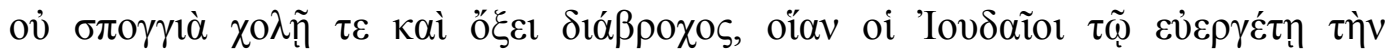

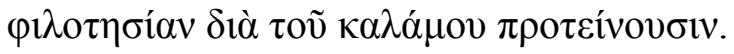

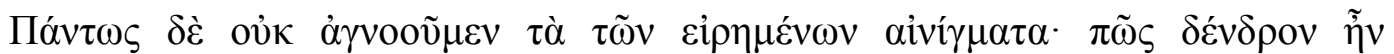

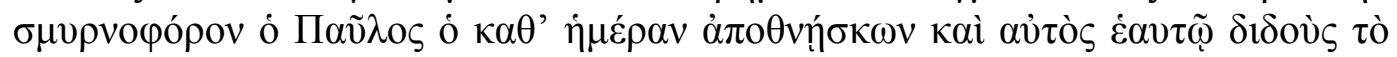

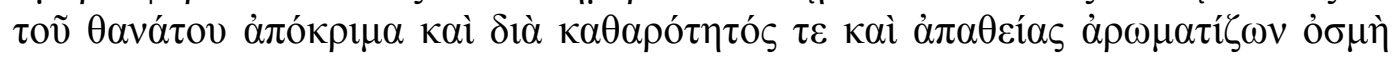

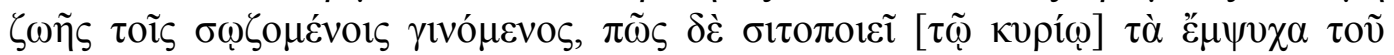

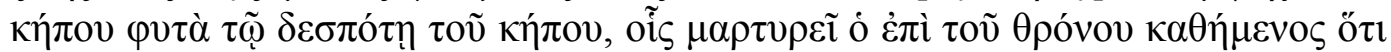

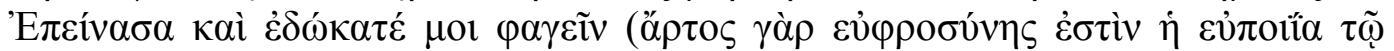

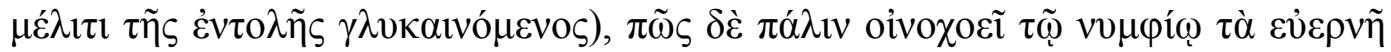

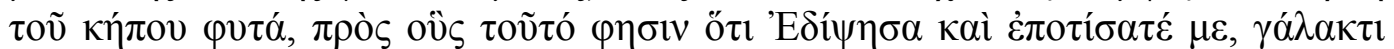

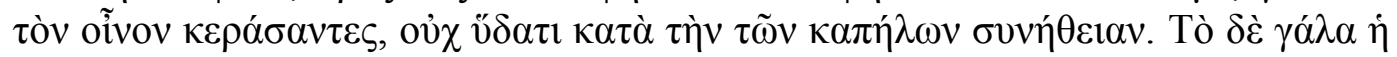

suite de la question, qui ne mentionne en aucun cas le miel. Les autres occurrences répertoriées par la Biblia patristica, III et V, Paris, 1980 et 1987, ne contiennent pas de citation de Lc 24, 42.

21 L'étude de J. A. Brooks, The New Testament Text of Gregory of Nyssa (The New Testament in the Greek Fathers 2), Atlanta, 1991, ne relève pas le verset de Lc 24, 42 et ne présente donc aucune analyse de la variante ; il ne traite pas non plus de Jn 21, 13 (voir infra).

22 Pour la date des Homélies, voir en particulier J. B. CAHILL, « The Date and Setting of Gregory of Nyssa's commentary on the Song of Songs ", Journal of Theological Studies 32, 1981, p. 447-460 ; F. DüNZL, "Gregor von Nyssa's Homilien zum Canticum auf dem Hintergrund seiner Vita Moysis », Vigiliae Christianae, 44, 1990, p. 371-381; A. CORTESI, Le "Omelie sul Cantico dei cantici" di Gregorio di Nissa: Proposta di un itinerario di vita battesimale (Studia ephemeridis augustinianum 70), Roma, 2000, p. 18. 


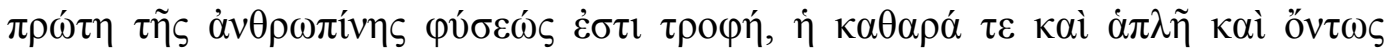

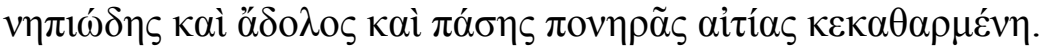

«Ô bienheureux ces jardins dont <l’Écriture> témoigne que les plantes produisent en abondance des fruits tels qu'ils se changent en toute espèce de délices de manière adaptée au désir d'en jouir (cf. Sg 16, 20-21)! Car pour celui qui fait ses délices de la bonne odeur, elles deviennent de la myrrhe avec des aromates $(\mathrm{Ct} 5,1 \mathrm{~b})$, parce qu'elles créent le parfum d'une vie pure et de bonne odeur, par l'état de mort des membres terrestres (cf. Col 3, 5), vie qui est un mélange des aromates variés et divers de la vertu, tandis que pour celui qui recherche la nourriture plus parfaite, elles deviennent $d u$ pain (cf. $\mathrm{Ct} 5,1 \mathrm{c}$ ), non plus celui qui se mange avec des herbes amères, comme le prescrit la Loi (cf. Ex 12,8) - car c'est pour le présent qu'est l'amertume - mais un pain qui se donne lui-même pour accompagnement du miel, lorsque le fruit de la vertu rend doux les sens de l'âme à son moment propre (cf. Ps 1,3) - le pain qui fut présenté aux disciples, assaisonné d'un rayon de miel, après la résurrection du Seigneur (cf. Jn 21,9 ; Lc 24, 42) en est la preuve 23 ; pour celui qui a soif, elles deviennent un cratère rempli de vin et de lait $(\mathrm{Ct} 5,1 \mathrm{~d})$, non une éponge imprégnée de fiel et de vinaigre comme la coupe d'amitié 24 que les Juifs tendirent à leur bienfaiteur au moyen d'un roseau (cf. Mt 27, 34.48).

Nous n'ignorons aucunement les énigmes de ces paroles : comment Paul était un arbre porteur de myrrhe, lui qui mourrait chaque jour (cf. 1 Co 15, 31), qui rendait lui-même contre lui-même la sentence de mort (cf. 2 Co 1,9) et qui, parce qu'il répandait un arôme de pureté et d'impassibilité, devenait une odeur de vie pour ceux qui étaient sauvés (cf. 2 Co 2, 15-16); comment les plantes animées du jardin produisent de la nourriture pour le Maître du jardin, elles à qui rend témoignage celui qui est assis sur son trône (cf. Mt 25, 31) : 'J'ai eu faim, et vous m'avez donné à manger' (Mt 25, 35) - car la bienfaisance est un pain de joie adouci par le miel du précepte (cf. Ps 18, 9.11) - et comment encore les plantes florissantes du jardin versent du vin à l'Époux, elles à qui il dit : 'J'ai eu soif, et vous m'avez donné à boire' (Mt 25, 35), puisqu'elles mêlent le vin au lait, et non à l'eau selon l'usage des cabaretiers (cf. Is 1, 22). Or le lait est le premier aliment de la nature humaine, pur, simple, vraiment adapté aux petits enfants, exempt de fraude (cf. 1 P 2, 2) et purifié de toute intention perverse. » 25

23 On suit la suggestion d'A. Rousseau (voir la traduction citée infra, n. **), p. 226, n. 1,

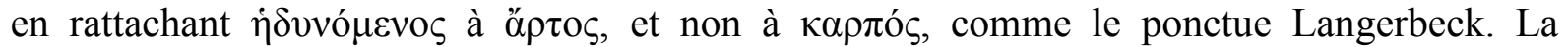
réflexion du traducteur, dans la même note, sur la difficulté d'identifier la source évangélique de Grégoire, qui fond ensemble deux passages évangéliques, ne lui a pas permis, cependant, d'effectuer le rapprochement avec l'autre texte de Grégoire étudié ci-dessous, non plus qu'avec Cyrille de Jérusalem.

24 Le terme est bien évidemment ironique.

25 Gregorre de Nysse, Homélies sur le Cantique, X (éd. H. Langerbeck [Gregorii Nysseni Opera VI], Leiden, 1960, p. 306, 8-308, 4). La présente traduction utilise comme point de départ celle d'A. RousSEAU (Donner raison 23), Bruxelles, 2008, p. 226-227, en la modifiant largement. Pour la thématique du lait comme aliment des petits enfants, voir la récente synthèse de J. D. Penniman, Raised on Christian Milk: Food and the Formation of the Soul in Early Christianity (Synkrisis: comparative approaches to early christianity in greco-roman culture), New Haven (CT), 2017. 
Cette explication des ingrédients de $\mathrm{Ct} 5,1 \mathrm{c}$ débouche ensuite sur une lecture typologique et spirituelle, appliquée à Paul puis au Maître du Jardin, le Christ. Il semble donc que, pour expliquer $\mathrm{Ct} 5,1 \mathrm{c}$, Grégoire reprenne et corrige l'explication de Cyrille de Jérusalem, en retenant certes l'interprétation liée à la Passion et à la résurrection, mais au milieu d'autres lectures du texte - ce qui se comprend dans le cadre d'une explication suivie du Cantique. Dans le contexte des apparitions du ressuscité, il substitue tacitement au poisson évangélique de Lc 24, qui ne correspond pas au texte du Cantique, le pain. Une telle démarche reposerait donc sur une fusion entre les aliments du bord du lac, (Jn 21, 9-14), pain et poisson, et ceux du repas à Jérusalem (Lc 24, 41-43), poisson et rayon de miel : on verra que le texte suivant de Grégoire confirme cette hypothèse. Le poisson, en quelque sorte mis en facteur commun entre les deux récits évangéliques, disparaît donc dans l'interprétation de Grégoire, faute de correspondant dans le texte du Cantique. Ne restent que le pain et le miel, qui à la fois trouvent leur correspondant vétérotestamentaire dans le texte du Cantique et sont interprétés du repas du ressuscité et de l'œuvre bienfaisante des hommes qui le suivent. Vin et lait de Ct $5,1 \mathrm{~d}$, enfin, sont opposés au fiel et au vinaigre de la Passion. La rapide allusion cyrillienne à l'opposition entre amertume et douceur, qui portait chez l'évêque de Jérusalem sur $\mathrm{Ct} 5,1 \mathrm{c}$, est reprise et développée par l'évêque de Nysse, avec entre autres un appui sur Ex 12, 8 (à propos de $\mathrm{Ct} 5,1 \mathrm{c}$ ), mais surtout en relation avec Mt 27, 34.48 qui sert à expliquer Ct 5, 1d. En effet, alors que Cyrille laissait de côté $\mathrm{Ct} 5$, 1d, vin et lait, Grégoire explique le stique et en fait le point d'appui principal de cette opposition entre amertume de la passion et douceur de la résurrection.

Il faut enfin introduire un deuxième texte nysséen, qui nous rapproche par un autre biais du texte de Cyrille de Jérusalem. Il s'agit en effet, cette fois, non d'un commentaire du Cantique mais d'un discours prononcé pour la Pâques, qui porte pour l'essentiel sur la question de l'intervalle entre la mort et la résurrection du Christ ; le contexte n'en est donc pas très éloigné de celui des catéchèses baptismales de Cyrille, qui trouvent place probablement à la fin du carême, voire au début de la Semaine sainte pour la Catéchèse $14^{26}$. Ce discours de Grégoire de Nysse, Sur l'intervalle de trois jours entre la mort et la résurrection $d u$ Christ, qui a été prononcé pendant la Vigile de la nuit pascale, doit être situé lui aussi assez tard dans la production nysséenne, probablement dans les mêmes années que les Homélies sur le Cantique 27 . À la fin du discours,

26 Pour la date liturgique des Catéchèses, et en particulier de la Catéchèse 14, voir S. GRIGNON, "La cohérence de la foi », cité n. **, p. 160-170, qui fournit la bibliographie antérieure; je remercie vivement S. Grignon pour m'avoir communiqué sa thèse inédite et éclairé sur cette question complexe.

27 Sur ce texte, voir en particulier Drobner, Gregor von Nyssa, Die drei Tage, cité n. ** (pour la date, voir p. 190-198); voir également, sur l'usage des récits de résurrection par Grégoire, en particulier dans ce discours, M. CASSIN, «Liturgical Celebration and Theological Exegesis: the Easter Homilies of Gregory of Nyssa », dans J. VERHEYDEN, 
pendant lequel il a étudié en particulier un certain nombre de questions disputées liées à la mort et à la résurrection du Christ, Grégoire introduit assez brusquement l'événement de la résurrection et le décrit avec des mots et des images tirés des évangiles, en une sorte de centon qui ne reflète exactement aucun des quatre évangiles, mais recompose librement le récit. Le dernier paragraphe du texte introduit plus proprement les auditeurs à l'eucharistie ; cette transition est élaborée avec soin, afin de lier les espèces eucharistiques aux éléments de la Pâque juive, pain sans levain et herbes amères, dont Grégoire venait de traiter dans la partie qui précédait 28 .

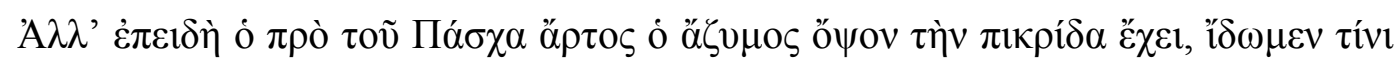

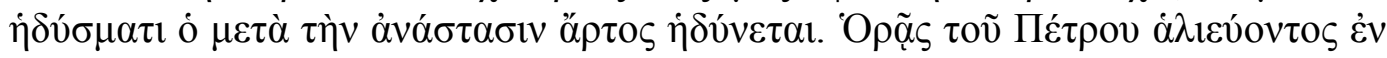

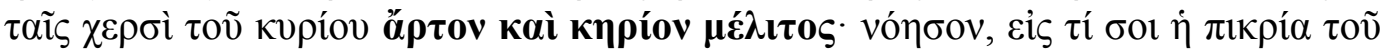

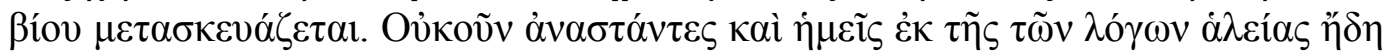

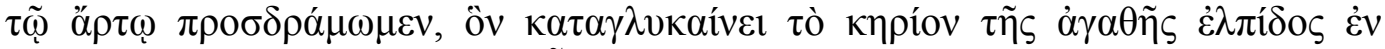
X

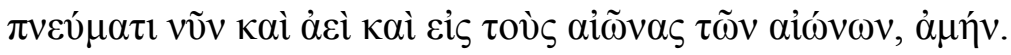

«Mais puisque le pain sans levain d'avant la Pâque a comme accompagnement les herbes amères, voyons par quel assaisonnement adoucir le Pain d'après la résurrection. Tu vois, tandis que Pierre est à la pêche, du pain et un rayon de miel dans les mains du Seigneur (cf. Jn 21, 3-14; Lc 24, 42) : comprends en quoi se transforme, pour toi, l'amertume de la vie. Donc, après nous être nous aussi relevés de la pêche des discours, courons immédiatement vers le Pain que rend doux le rayon de miel de la bonne espérance dans le Christ Jésus notre Seigneur : à lui la gloire et la puissance, avec le Père et le Saint-Esprit, maintenant et toujours et dans les siècles des siècles. Amen. »29

Dans ce discours, contrairement à ce qui se passait dans les Homélies sur le Cantique, l'intégration du miel dans l'épisode de Jn 21 est explicite, tandis que Ct $5,1 \mathrm{c}$, en revanche, a disparu. Nous nous trouvons donc dans la situation symétrique à la précédente : le cadre principal est celui de la pêche au bord du lac, non du repas à Jérusalem, de Jean, non de Luc. Ct 5, 1 n'est pas explicitement présent, ni ici, ni plus haut dans le texte. L'insertion liturgique, elle, est explicite, puisque Grégoire invite à passer de la pêche des discours (liturgie de la parole, que clôt sûrement son discours, c'est-à-dire son homélie) au Pain eucharistique. On retrouve toutefois la transition de l'amertume du récit de l'Exode à la douceur de la Pâques chrétienne, qui était présente en deux

A. MERKT et T. Nicklas (éd.), "If Christ has not been raised... " Studies on the Reception of the Resurrection Stories and the Belief in the Resurrection in the Early Church (Novum Testamentum et Orbis Antiquus / Studien zur Umwelt des Neuen Testaments 115), Göttingen, Bristol (CT), 2016, p. 149-165.

28 Gregoire de Nysse, De tridui spatio (éd. E. Gebhardt, dans G. Heil, A. VAn Heck, E. GEBHARDT et A. SPIRA, Gregorii Nysseni Sermones I [Gregorii Nysseni Opera IX], Leiden, 1967, p. 271-306), p. 295, 21-296, 22.

29 GREGOIRE DE NYSSE, De tridui spatio, p. 306, 1-10. Je remercie Jean Reynard, qui m'a aimablement communiqué sa traduction, à paraître dans la collection Sources chrétiennes ; je l'ai librement adaptée ici. 
étapes dans les Homélies sur le Cantique, d'abord à propos du pain $(\mathrm{Ct} 5,1 \mathrm{c})$ puis du vin et du lait (Ct 5, 1d). Ici, l'évêque de Nysse avait besoin de pain et de miel, pour répondre terme à terme au pain et aux herbes amères de la Pâques juive qu'il avait évoqués précédemment. Il avait également besoin du pain pour marquer de manière claire la transition entre son discours et le repas eucharistique qui le suivait, dont le repas au bord du lac avec le Christ ressuscité figure un type. Grégoire de Nysse déplace donc la variante de Lc 24, 42 en Jn 21, 13 ; elle est ainsi introduite dans un contexte général similaire - un repas du ressuscité - mais différent, puisqu'il ne s'agit plus de Jérusalem, mais du lac de Tibériade, qu'il ne s'agit plus du récit de $L u c$, mais de celui de Jean. Ce déplacement permet en outre à Grégoire d'améliorer l'adéquation proposée par Cyrille de Jérusalem entre $\mathrm{Ct} 5,1 \mathrm{c}$ et le repas du ressuscité, mais aussi entre le pain accompagné d'herbes amères de l'Exode et le pain et le miel du ressuscité. Cette meilleure adéquation n'est toutefois atteinte qu'au prix d'une fusion des deux récits de repas avec le ressuscité. En outre, le cadre johannique est ici mieux adapté, dans la mesure où dans ce repas au bord du lac, c'est Jésus qui invite ses disciples à manger ce qu'il a préparé, situation qui convient davantage au repas eucharistique que le modèle lucianique où Jésus demande à ses disciples de lui donner quelque chose à manger. On relèvera enfin que les deux textes de Grégoire témoignent l'un et l'autre de leur filiation à la catéchèse de Cyrille, le premier par l'intermédiaire du verset du Cantique, l'autre par le contexte pascal et baptismal du discours.

Il est tentant de voir dans le rapprochement de ces deux textes nysséens une aide possible pour dater le discours Sur l'intervalle de trois jours; en effet, il semble bien que les Homélies sur le Cantique fournissent une première reprise du modèle fourni par Cyrille de Jérusalem pour expliquer Lc 24, 42var., où Ct 5, 1c est central. Au contraire, dans le discours Sur l'intervalle de trois jours, Ct 5, $1 \mathrm{c}$ ne figure pas, mais la fusion des deux repas lucanien et johannique apparaît plus aboutie. On pourrait donc être tenté de pousser un peu plus loin les remarques éparses d'H. R. Drobner dans son étude et de situer le discours après les Homélies sur le Cantique 30 ; il faut cependant rester prudent et faire la part de la différence de genre littéraire, de contexte et de public entre les deux textes. Il paraît assuré, cependant, que ce rapprochement confirme la date tardive du discours Sur l'intervalle de trois jours.

Chez Cyrille de Jérusalem comme chez Grégoire de Nysse, la forme longue de Lc 24, 42 est donc perçue comme la forme normale du texte et ne suscite aucune tentative de justification, y compris dans la prédication : c'est le texte reçu et connu de tous, non une variante au statut marginal. Dans les deux cas, elle est utilisée en lien avec $\mathrm{Ct} 5,1 \mathrm{c}$ et en lien typologique avec le repas pascal de l'Exode, ce qui explique la transposition de la mention du rayon de miel dans le récit johannique au bord du lac. Aucun des deux auteurs ne commente le poisson, présent pourtant dans les deux repas ; on le comprend aisément dans la

30 DroBnER, Gregor von Nyssa, Die drei Tage, cité n. **, p. 190-198 et passim. 
mesure où ils ne proposent pas un commentaire suivi du récit évangélique, mais l'utilisent simplement dans un contexte de prédication ou de commentaire d'un autre texte scripturaire. En outre, d'un point de vue typologique, pain et miel présentent un intérêt beaucoup plus immédiat, que ce soit en lien avec le Cantique ou avec l'Exode.

\section{III. - AUTRES TEMOINS PATRISTIQUES GRECS DE LC 24, 42 FORME LONGUE}

Qu'en est-il des autres témoins patristiques de Lc 24, 42 forme longue ? Ils ne sont pas très nombreux dans le monde grec (davantage dans le monde latin); pour l'essentiel, ils sont postérieurs, ou de très peu antérieurs, aux textes de Cyrille de Jérusalem et de Grégoire de Nysse que nous venons d'étudier; surtout aucun, dans le monde grec, ne fait un usage explicite du miel et aucun autre que les textes mentionnés ne rapproche le verset de $L u c$ de celui du Cantique. La plupart des citations de la forme longue se trouvent dans le contexte des discussions sur la corporéité du Christ ressuscité. On la trouve par exemple chez Épiphane de Salamine ${ }^{31}$, dans la notice du Panarion contre les manichéens ${ }^{32}$. L'ouvrage a été composé dans les années 370 et prend donc place après les citations cyrilliennes, mais avant celles de Grégoire de Nysse. La définition du type de texte utilisé par Épiphane ne va pas sans difficulté 33 : en fonction des livres, on a relevé des affinités avec un texte égyptien tardif, mais avec également une influence du texte byzantin, ce qui pourrait tout à fait correspondre à la situation que l'on relève pour Lc 24, 42 .

On trouve également une citation de Lc 24, 42var. cher Amphiloque d'Iconium ${ }^{34}$, dans son traité contre les encratites, qui remonte probablement au

31 Sur l'auteur et son œuvre, voir en particulier Y. R. KIM, Epiphanius of Cyprus. Imagining an Orthodox World, Ann Arbor (MI), 2015, et A. S. JACOBS, Epiphanius of Cyprus: a cultural Biography of Late Antiquity (Christianity in Late Antiquity 2), Oakland (CA), 2016.

32 ÉpIPhane de SAlamine, Panarion, 66, 39, 1-3 (éd. K. Holl, revue par J. Dummer

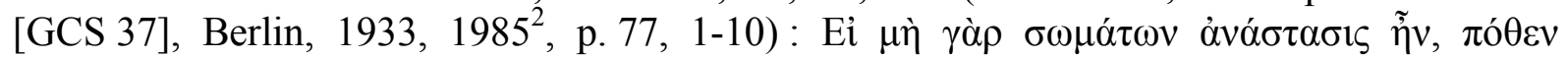

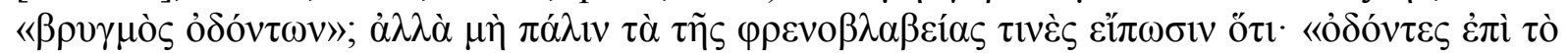

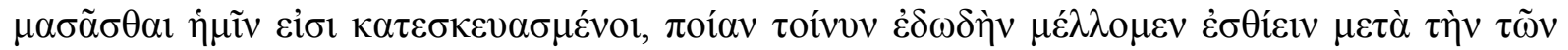

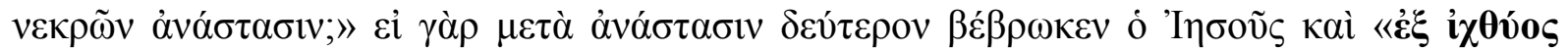

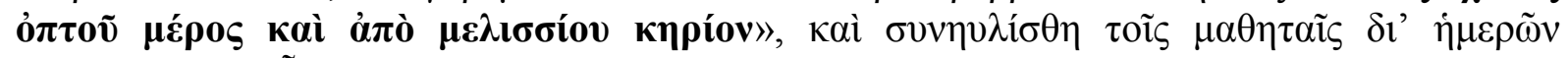

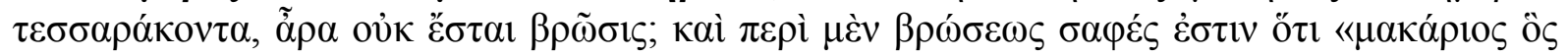

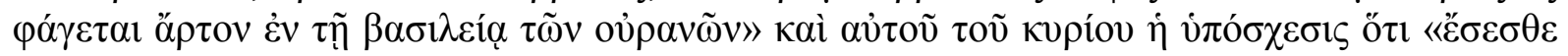

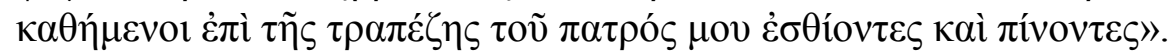

33 Pour le type (ou les types) de texte néo-testamentaire utilisé(s) par Épiphane, voir L. A. EldRIDGE, The Gospel Text of Epiphanius of Salamis (Texts and Documents 41), Salt Lake City, 1969 (non uidi), et surtout C. OSBURN, The Text of the Apostolos in Epiphanius of Salamis (The New Testament in the Greek Fathers 6), Leiden, 2004.

34 Sur Amphiloque et son œuvre, voir en particulier l'introduction d'A. SEGNERI, La controversia trinitaria del IV secolo nell'esegesi dottrinale di Anfilochio di Iconio (Studia ephemeridis Augustinianum 147), Roma, 2016, p. 15-59, qui fait le point sur les œuvres et leur authenticité. 
dernier quart du $4^{\mathrm{e}}$ siècle 35 : la citation intervient à la fin d'un chapitre consacré au témoignage de $L u c$ sur le comportement de Jésus après la résurrection, qui vise à prouver la réalité corporelle de la résurrection, entre autres par l'usage que le Christ fait des fruits de la création. L'auteur, cependant, ne commente pas les différents aliments. Plus tard encore, Cyrille d'Alexandrie, dans son Commentaire sur Jean, cite le verset de Luc, y compris la mention du rayon de miel, mais ne commente que la mention du poisson, dont il fait un témoignage de la résurrection du Christ dans la chair ${ }^{36}$.

Ce sont cependant des textes de datation plus difficiles qui retiendront ici notre intérêt: en effet, on retrouve la forme longue de Lc 24, 42 dans le Quatrième discours contre les ariens, attribué à Athanase, dans un passage contre Paul de Samosate 37 . Or les études sur ce texte suggèrent d'en situer la rédaction peu avant 340 , dans le contexte d'une tentative de rapprocher les positions d'Eusèbe de Césarée et de Marcel d'Ancyre. M. Vinzent a suggéré d'attribuer le texte à Apolinaire, sans que cette attribution ait été pour autant

35 Amphiloque D'ICOnium, Contra haereticos, 27 (éd. C. Datema [Corpus christianorum, Series graeca 3], Turnhout, Leuven, 1978, 1. 1054-1058): Oi $\delta \dot{\varepsilon} \varepsilon \dot{\varepsilon} \pi \varepsilon ́ \delta \omega \kappa \alpha v$

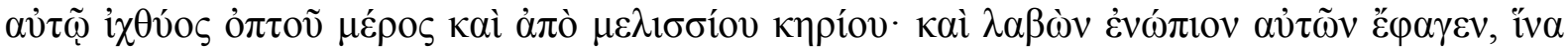

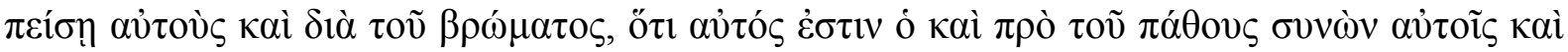
$\sigma v v \delta 1 \alpha \iota \tau \omega \mu \varepsilon v o \zeta$. Sur ce texte, voir en particulier F. AMSLER, « Amphiloque d'Iconium, Contre les hérétiques encratites et apotactites. Traduction française », dans A. FREY, R. GOUNELLE (éd.), Poussières de christianisme et de judaïsme antiques. Études réunies en l'honneur de Jean-Daniel Kaestli et Éric Junod, Lausanne, 2007, p. 7-40.

36 Cyrille D'AleXANDrie, In Iohannem, XII (éd. Ph. E. PuSEY, Oxford, 1872, III, p. 150,

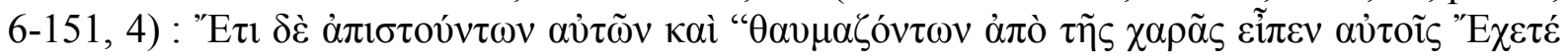

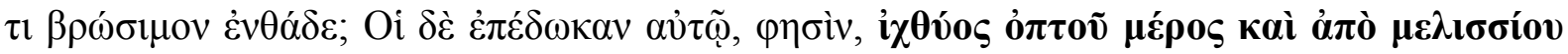

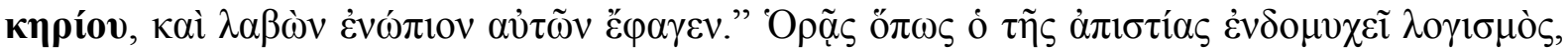

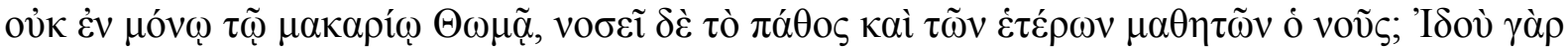

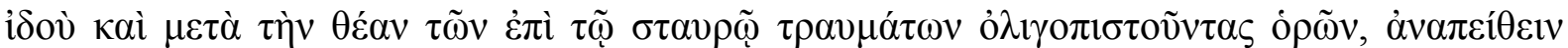

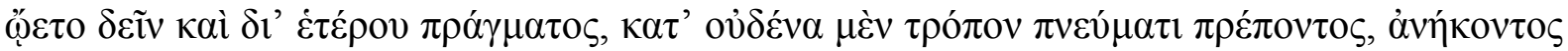

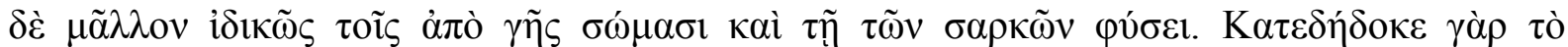

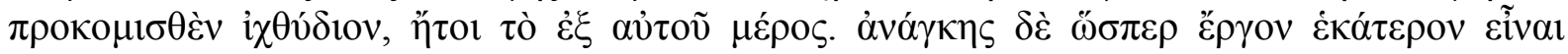

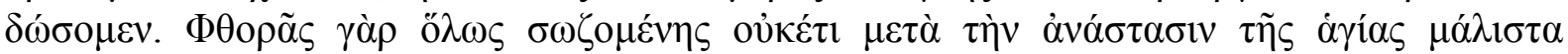

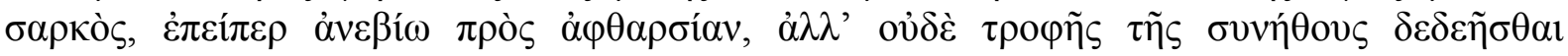

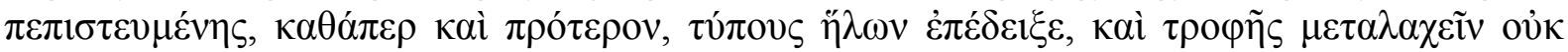

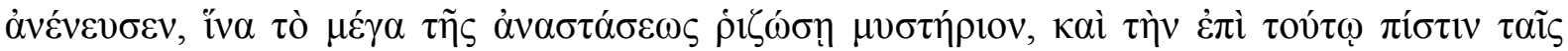

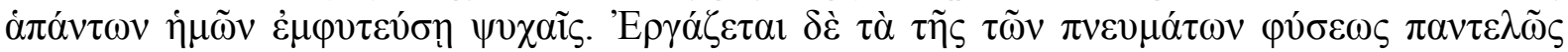

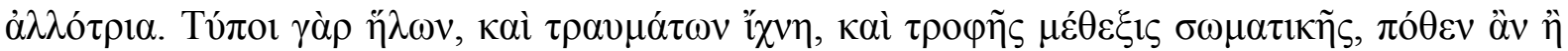

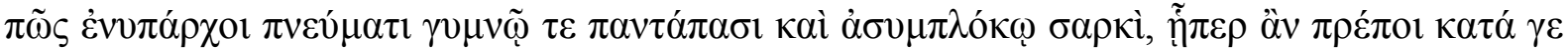

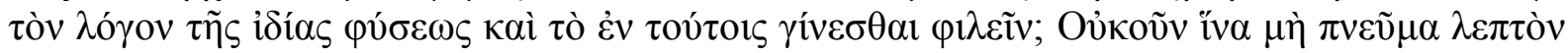

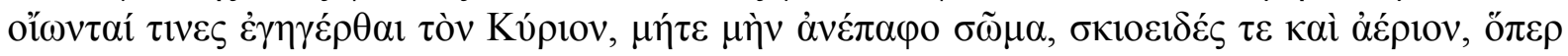

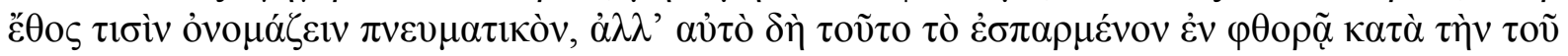

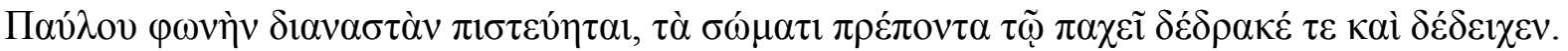

37 Sur ce texte, voir en dernier lieu M. VInZENT, Pseudo-Athanasius, Contra Arianos IV. Eine Schrift gegen Asterius von Kappadokien, Eusebius von Cäsarea, Markell von Ankyra und Photin von Sirmium (Supplements to Vigiliae Christianae 36), Leiden, New York, Köln, 1996. 
considérée comme acquise. Si la datation est juste, cependant, il s'agirait sans doute du premier témoin patristique explicite de la forme longue 38 .

En effet, l'autre texte qui est cité comme un témoignage de cette variante provient du traité du pseudo-Justin Sur la résurrection ${ }^{39}$; or ce texte ne nous est connu que de manière fragmentaire, par l'intermédiaire de florilèges, en particulier les Hiéra, traditionnellement attribués à Jean Damascène ${ }^{40}$. Le texte de Lc 24, 42 n'y est pas cité, mais paraphrasé, d'une manière qui pourrait exclure une interpolation postérieure à partir du texte byzantin reçu - ce qui reste cependant tout à fait possible étant donné que nous ne connaissons plus de l'œuvre que des extraits transmis par un florilège, c'est-à-dire dans un contexte de circulation qui n'exclut pas, au contraire, la réécriture 41 . Les études récentes ont proposé de situer le texte du pseudo-Justin, dont l'origine et la date restent très débattues, vers la fin du $2^{\mathrm{e}}$ siècle. Cependant, étant donné l'incertitude sur l'origine et la datation du texte et la multitude d'hypothèses émises à cet égard, il est difficile d'utiliser ce témoignage pour préciser l'origine tant chronologique que géographique de la variante ${ }^{42}$. Même si les études récentes ont proposé de

38 Pseudo-Athanase, Quatrième discours contre les ariens, 35, 23-40 (éd. A. Stegmann, Die pseudoathanasianische 'IVte Rede gegen die Arianer' als ' $\kappa \alpha \tau \hat{\alpha}$ A $\rho \varepsilon \imath \alpha v \tilde{\omega} v$

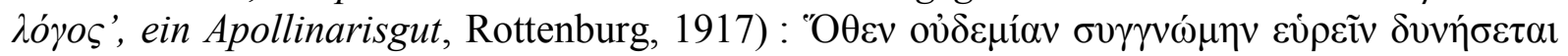

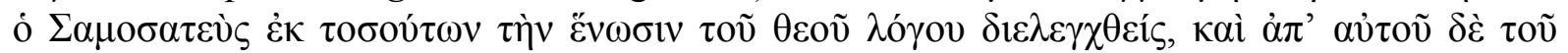

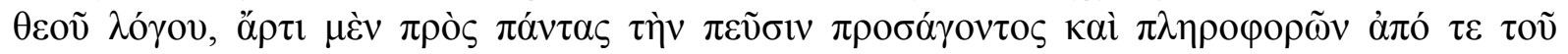

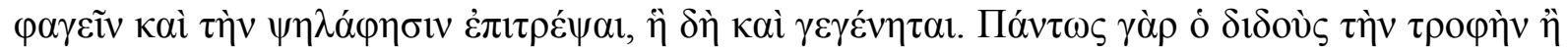

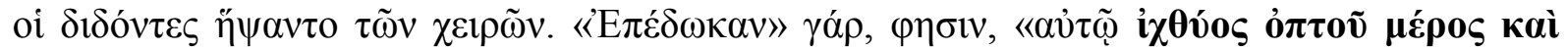

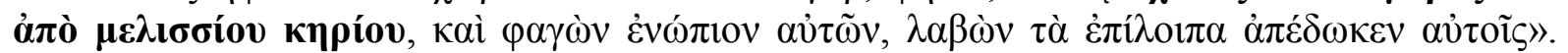

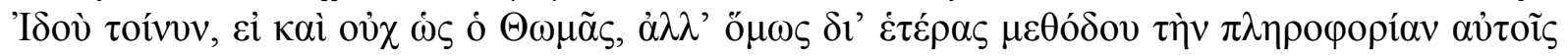

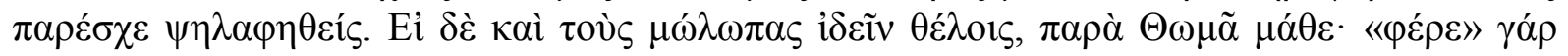

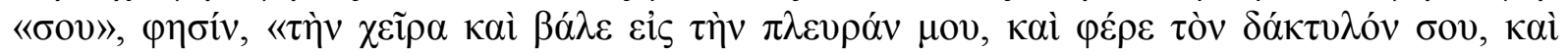

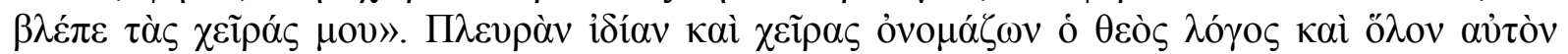

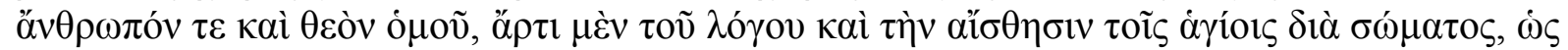

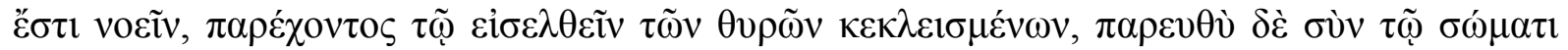

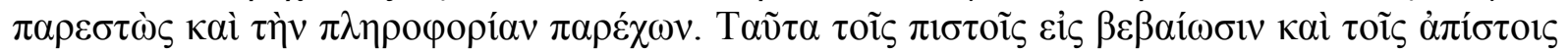

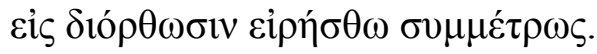

39 Voir M. HeIMgartner, Pseudo-Justin - Über die Auferstehung, Text und Studie (Patristische Texte und Studien 54), Berlin, New York, 2001 ; A. D’AnNA, Pseudo-Giustino, Sulla resurrezione, Discorso cristiano del II secolo (Letteratura cristiana antica), Brescia, 2001.

40 Pour la date et l'attribution du florilège, voir en dernier lieu J. DECLERCK, « Les Sacra Parallela nettement antérieurs à Jean Damascène », Byzantion 85, 2015, p. 27-65.

41 Pseudo-Justin, Sur la résurrection, 9, 7 (éd. HeImgARTNER, cité n. **, p. 124-125) :

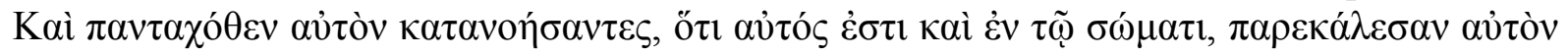

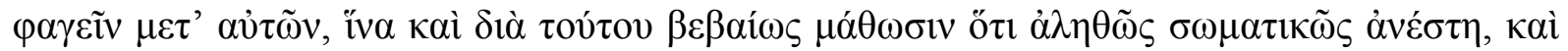

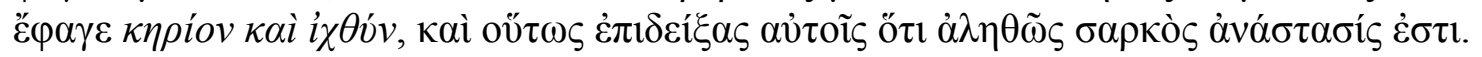

42 L'édition critique des Sacra parallela n'est pas encore disponible pour le livre I, dans lequel figure 1'extrait du pseudo-Justin; voir T. THUM, Die Schriften des Johannes von Damaskos. VIII, Sacra (spuria). 4, Liber II, De rerum humanarum natura et statu. Erste Rezension. Ertser Halbband. A-E (II'1-1000) (Patristische Texte und Studien 74), Berlin, 
voir dans le texte du pseudo-Justin la première attestation de la forme longue de Lc 24, 42, ce qui n'est pas impossible, la prudence s'impose dans la mesure même où ce témoignage est isolé et le seul à remonter clairement avant le $4^{\mathrm{e}}$ siècle 43 . Il faudrait à la fois pouvoir assurer que le texte d'origine, avant son insertion dans le florilège, contenait bien la forme longue de Lc 24, 42, et préciser la date et l'origine de ce texte pseudo-justinien, afin de pouvoir en tirer pleinement parti pour comprendre d'où vient cette mention du rayon de miel.

Il serait trop long de présenter ici en détail tous les témoignages latins ; qu'il suffise de rappeler qu'il n'y a pas de citation du verset sous sa forme longue avant Jérôme et Augustin dans le monde latin 44 , du moins si l'on écarte, comme j'ai proposé de le faire au début de cet article, à la suite de bien d'autres, le témoignage supposé de Tertullien dans le De corona. Il n'est pas question non plus de reprendre ici l'enquête sur la symbolique du miel dans les représentations de la Terre promise et de la résurrection, non plus que le dossier de Joseph et Aseneth et l'usage qui y est fait du miel ${ }^{45}$, afin de tenter d'évaluer les origines symboliques et liturgiques de la variante. Je laisserai volontiers à d'autres cette enquête, qui échappe largement au champ textuel pour entrer dans le domaine de l'histoire des religions et de l'anthropologie.

$\mathrm{Au}$ terme de cette brève enquête, il apparaît donc que la forme longue de Lc 24, 42 n'est pas attestée de manière sûre avant le deuxième tiers du $4^{\mathrm{e}}$ siècle

2018 ; IDEM, 5, Liber II, De rerum humanarum natura et statu. Erste Rezension. Zweiter Halbband. Z-Omega (II'1001-2293) (Patristische Texte und Studien 75), Berlin, 2018; J. DeClERCK, 6, Liber II (De rerum humanarum natura et statu), Zweite Rezension/Erster Halbband, Berlin, Boston, 2018 ; IDEM, 7, Liber II (De rerum humanarum natura et statu), Zweite Rezension/Zweiter Halbband, Berlin, Boston, 2018.

43 M. Heimgartner, Pseudo-Justin, cité n. **, adopte une position ambigüe; dans son commentaire du passage (p. 180, n. 204 $)$, il relève que les attestations explicites ne remontent pas avant le $4^{\mathrm{e}}$ siècle, mais renvoie cependant au passage du Pédagogue de Clément d'Alexandrie (voir supra, p. **), dont l'étude menée dans l'introduction de l'ouvrage (p. 8586) tend à faire un témoin indirect de la variante longue. A. D'ANNA, Pseudo-Giustino, cité n. **, p. 49 et n. 55, considère la variante comme bien attestée, en renvoyant au Diatessaron par l'intermédiaire de l'apparat de l'édition néotestamentaire du Greek New Testament, Stuttgart, 1983 ; sur le témoignage du Diatessaron et la difficulté qu'il y a à reconstituer l'état d'origine de ce texte en ce point, voir supra, p. ***. Le texte du pseudo-Justin est également considéré comme un témoignage fiable par CHERUBINI, "Mangiò pesce e miele", cité n. **, p. 27-28.

44 Voir la liste des textes et leur analyse dans CHERUBINI, "Mangiò pesce e miele”, cité n. ${ }^{* *}$, p. 46-64, ainsi que sa présentation d'autres témoignages latins, tous postérieurs. Ce constat corrobore le relevé fait pour les manuscrits latins du Nouveau Testament (voir supra, p. $\left.{ }^{* *}\right)$ : la variante longue ne se diffuse dans le monde latin qu'à partir de la fin du $4^{\mathrm{e}}$ siècle.

45 Sur ce texte, voir en dernier lieu l'édition traduite et commentée dirigée par E. REINMUTH, Joseph und Aseneth (SAPERE 15), Tübingen, 2009, ainsi que celle dirigée par Ch. Burchard, Joseph und Aseneth (Pseudepigrapha Veteris Testamenti Graece 5), Leiden, 2003. 
- réserve faite du texte problématique et isolé du pseudo-Justin. En outre, ses premières attestations se trouvent en Syrie-Palestine et en Asie mineure. Rares sont les auteurs grecs de l'époque patristique à avoir proposé une interprétation de cette forme du texte dans le monde grec. Lorsqu'elle existe, et ce seulement chez Cyrille de Jérusalem et Grégoire de Nysse, elle s'appuie sur un rapprochement avec $\mathrm{Ct} 5,1 \mathrm{c}$, qui mentionne pain et miel. Elle est en outre motivée par une opposition, plus courante, entre l'amertume de la passion, soutenue par la mention du vin mêlé de fiel proposé à Jésus avant sa mise en croix, et la douceur de la résurrection, figurée par le miel. Cette opposition est présentée de manière typologique en lien avec l'opposition vétérotestamentaire entre les herbes amères du repas pascal et le lait et le miel de la Terre promise. Si Cyrille de Jérusalem et Grégoire de Nysse reprennent cette opposition et l'appliquent à la forme longue de Lc 24, 42, toute utilisation de l'opposition entre fiel et miel n'implique pas une référence à Lc 24, 42var.

En second lieu, on n'a pu repérer aucun lien explicite entre Lc 24, 42var. et la pratique qui consistait à donner du lait et du miel aux nouveaux baptisés; au contraire, dans le discours pascal de Grégoire de Nysse, c'est du pain eucharistique que sont rapprochés pain et miel, et non d'une nourriture spécifique au nouveau baptisé. Il semble donc que, pour les auteurs du $4^{\mathrm{e}}$ siècle, la mention du miel aux côtés du poisson grillé en Lc 24, 42, n'est pas mise en relation avec une pratique liturgique ; c'est à partir de $\mathrm{Ct} 5,1 \mathrm{c}$ et de l'opposition entre amertume de la Passion et douceur de la résurrection qu'ils cherchent à expliquer le passage. Si, culinairement, l'origine de la variante est compréhensible - du poisson avec une sauce au miel, ou suivi d'un rayon de miel - ses origines réelles restent obscures. Il demeure difficile de la situer chronologiquement - même si aucune attestation incontestable n'en est conservée avant le milieu du $4^{\mathrm{e}}$ siècle - et géographiquement - bien que les indices convergent, grossièrement, vers la Syrie-Palestine et l'Asie mineure. Elle n'a que rarement retenu l'attention des exégètes antiques, en particulier dans le monde hellénophone, peut-être parce qu'elle leur paraissait plus anodine qu'à nous, ou parce qu'elle ne figure que chez des auteurs qui n'ont que peu d'intérêt pour la critique textuelle.

Matthieu CASSIN

IRHT (CNRS, Paris) 